\title{
Características nutricionales de genotipos de quinoa del nivel del mar cultivadas en ambientes templados de Argentina
}

\author{
Sanahuja, M.C. • Vidueiros, S.M. • Bertero H.D. • Pallaro A.
}

Para citar este artículo: Sanahuja, M.C.; Vidueiros, S.M.; Bertero H.D.; Pallaro A.. (2019). Características nutricionales de genotipos de quinoa del nivel del mar cultivadas en ambientes templados de Argentina. Pág. XX-XX. Santa Fé, Argentina: UNL. DOI 10.14409/fabicib.v23i0.7885 


\section{CARACTERÍSTICAS NUTRICIONALES DE GENOTIPOS DE QUINOA DEL NIVEL DEL MAR CULTI- VADAS EN AMBIENTES TEMPLADOS DE ARGENTINA}

Sanahuja, M.C. ${ }^{1}$; Vidueiros, S.M. ${ }^{1}$; Bertero H.D. ${ }^{2}$; Pallaro A. ${ }^{1}$

mcsanahuja@ffyb.uba.ar

${ }^{1}$ Universidad de Buenos Aires. Facultad de Farmacia y Bioquímica. Cátedra de Nutrición. Junín 956. 2o piso. CP 1113. CABA. Argentina. Tel: 541152874208

${ }^{2}$ Universidad de Buenos Aires. Facultad de Agronomía. Departamento de Producción Vegetal. Av. San Martin 4453. CP1417. CABA. Argentina

Recibido: 04/01/2019. Aceptado: 11/11/2019

\section{RESUMEN}

La composición nutricional de la quinoa proveniente de las regiones tradicionales de cultivo es bien conocida, sin embargo es necesario investigar las propiedades nutricionales de aquella cultivada en otras regiones. El objetivo de este trabajo fue estudiar composición centesimal, perfil de ácidos grasos y contenido total de saponinas de 4 genotipos de quinoa del nivel del mar (NM) cultivadas en ambientes templados de Argentina y compararlas con las de quinoa nativa del noroeste argentino (NOA), que fuera previamente estudiada. El contenido proteico fue significativamente menor (NM: 12.8 vs NOA: $16.8 \mathrm{~g} 100^{-1} \mathrm{~g} ; \mathrm{p}<0.0001$ ), mientras que el de ácidos grasos poliinsaturados fue mayor (NM: 87.8 vs NOA: 83\%; p<0.001), a expensas del ácido linoleico. Asimismo, todos los atributos nutricionales se encontraron dentro de los rangos reportados en la literatura internacional. Los resultados obtenidos sugieren que el genotipo y el ambiente de cultivo incidirían en las propiedades nutricionales de la quinoa, lo que debería ser tenido en cuenta para su elección y aprovechamiento desde el punto de vista nutricional. Conocer las diferencias entre quinoas de distintas regiones de Argentina contribuye con datos nacionales en las tablas de composición de alimentos.

Palabras claves: quinoa; genotipo nivel del mar; características nutricionales 


\section{SUMMARY}

\section{Nutritional traits of sea level quinoa genotypes cultivated in temperate environments of Ar- gentina}

The nutritional composition of quinoa from traditional growing regions is well known, however it is necessary to investigate the nutritional traits of that cultivated in other regions. The objective of this work was to study the proximal composition, the fatty acid profile and the total saponin content of 4 genotypes of sea level quinoa (SL) cultivated in temperate environments of Argentina and to compare them with those of native quinoa of northwestern Argentina (NWA), which was previously studied. The protein content was significantly lower (SL: 12.8 vs. NWA:

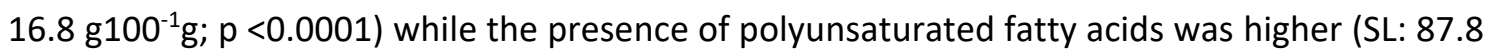
vs. NWA: $83 \% ; p<0.001$ ), in terms of linoleic acid. However, all the nutritional attributes were found within the ranges reported in the international literature. The results obtained suggest that the genotype and the growing environment would affect the nutritional properties of quinoa, which should be taken into account for their selection and use from the nutritional point of view. Knowing the differences between quinoa from different regions of Argentina contributes with national data in the tables of food composition.

Keywords: quinoa: sea level genotype; nutritional traits

\section{INTRODUCCIÓN}

La quinoa (Chenopodium quinoa Willd.), de la familia de las Amaranthaceae, es uno de los granos más antiguos del continente americano. Su origen se sitúa hace varios miles de años en las tierras altas de la región andina (1). La gran diversidad genética de este cultivo le permite adaptarse a diversos tipos de suelo, y a una amplia gama de humedad y temperatura para su cultivo (2-4). De acuerdo con esto, se lo clasifica en 5 ecotipos: altiplano, salares, valles interandinos, subtropical y nivel del mar (5-9). La quinoa a nivel del mar se cultiva a baja altura (10) y su baja sensibilidad al fotoperíodo la hace apta para el cultivo en ambientes templados (11). La del altiplano, en cambio, llega a cultivarse hasta a 3800 msnm en condiciones de frío seco y de gran amplitud térmica durante el día (7).

Este antiguo cultivo andino se ha convertido en un grano de interés a los fines de mejorar la diversidad de las dietas occidentales dado su valor nutricional principalmente relacionado a su alto contenido de proteínas, minerales y vitaminas, comparado con el de los cereales y otros granos (1-4, 1217). 
Se ha reportado que la composición nutricional de la quinoa varía entre los distintos ecotipos debido a la fuerte variabilidad genética y a las diferencias ambientales en las distintas regiones de cultivo (18-25), siendo el ecotipo del altiplano el más estudiado.

La Argentina por su extensión se caracteriza por poseer distintas regiones geográficas, con diversos climas, altitudes, tipos y salinidad de los suelos. Dada la adaptabilidad que caracteriza a este grano, en la actualidad está siendo cultivada en diferentes regiones de nuestro país, estando su cultivo particularmente difundido en la zona del noroeste (NOA) y parte de la Patagonia andina, Neuquén y Chubut.

En estudios previos, se evaluaron las características nutricionales de 21 poblaciones de quinoa originarias del NOA recolectadas en diferentes ecorregiones de las provincias de Salta y Jujuy, lo que permitió la valorización del germoplasma nativo de quinoa del NOA frente a cultivos de otros orígenes (23).

Por otra parte, dado que en la región pampeana de nuestro país la agricultura está tendiendo a la utilización de sistemas de uso más intensivo de la tierra y labranza reducida, los diferentes genotipos de quinoa del nivel del mar se presentan como una alternativa interesante a los cereales de invierno (26). Aunque las propiedades nutricionales de la quinoa de las regiones tradicionales son bien conocidas, es necesario caracterizar la quinoa cultivada en diferentes regiones de Argentina para generar conocimiento sobre su valor nutricional y obtener datos nacionales de las producciones locales lo que podría favorecer el desarrollo de las economías regionales.

Por lo tanto, el objetivo de este trabajo fue estudiar las características nutricionales de genotipos de quinoa del nivel del mar cultivadas en ambientes templados y evaluar comparativamente las mismas con las del germoplasma nativo del noroeste argentino.

\section{MATERIALES Y MÉTODOS}

\section{Material}

\subsection{Semillas de quinoa}

Se caracterizaron 4 genotipos de quinoa, adaptados a ambientes templados, que se multiplicaron en una estación experimental en la Facultad de Agronomía de la Universidad de Buenos Aires (34ㅇ's, 5929' O; 20 msnm) en suelo de tipo arcilloso limoso (vertic argiudoll, Taxonomía USDA). Los genotipos evaluados fueron: Salto de Agua (Chile), CO-407(EEUU), NL-6 (Holanda), y 2-Want (EEUU). Los 3 primeros se seleccionaron a partir de germoplasma proveniente de quinoas de ambientes de baja altitud 
de Chile (llamados quinoas del nivel del mar) (27), mientras que 2- Want es el resultado del cruzamiento entre una accesión boliviana y una chilena (E. Ballon, comunicación personal).

La siembra se realizó en parcelas ordenadas de forma aleatoria con diseño de bloque completo con tres réplicas. En las mismas, se plantaron a mano 20 plantas por $\mathrm{m}^{2}$ en hileras a $0.50 \mathrm{~m}$ de distancia una de la otra. Las parcelas eran de cinco filas de ancho por $3 \mathrm{~m}$ de largo con una superficie de $7,5 \mathrm{~m}^{2}$. Las plantas recibieron irrigación y fertilización suplementaria en siembra (20 kg P y $18 \mathrm{~N} \mathrm{~kg} \mathrm{ha}^{-1}$ ) y una aplicación de urea (total de $100 \mathrm{~kg} \mathrm{~N} \mathrm{ha}^{-1}$ ) 30 días después de la germinación para minimizar las restricciones de nutrientes. Las malezas fueron removidas a mano y se aplicaron fungicidas e insecticidas cuando se detectaron enfermedades y plagas.

\section{Métodos}

\subsection{Composición centesimal}

La composición centesimal se determinó sobre las semillas de quinoa crudas siguiendo la metodología especificada por la Association of Official Analytical Chemist (AOAC) (28), humedad por AOAC no 925.09, cenizas por AOAC no 923.03, proteínas por AOAC no 984.13 y grasa por AOAC no 930.09. El factor utilizado para transformar \% de nitrógeno en proteínas fue $6.25(3,23)$. El contenido de fibra dietaria total (FDT) se determinó sobre muestras secas y desengrasadas utilizando AOAC nำ85.29 adoptado por el kit comercial Megazyme ${ }^{\circledR}$. El porcentaje de carbohidratos se calculó con la siguiente fórmula:

$\%$ Carbohidratos $=100-(\%$ humedad $+\%$ cenizas $+\%$ proteínas $+\% g r a s a+$ fibra dietaria $)$

\subsection{Perfil de ácidos grasos}

La extracción de los lípidos totales de las semillas de quinoa se realizó utilizando la mezcla de Folch (29) (cloroformo: metanol 2:1, v/v). Después de la eliminación de colesterol y lípidos, cuya presencia interfiere con el método de análisis, la fase de cloroformo se trató con una solución trifluoruro de boro al $10 \%$ en metanol en atmósfera de nitrógeno, durante 30 minutos a $80^{\circ} \mathrm{C}$. Mediante este procedimiento se transforman los ácidos grasos en sus derivados más volátiles que son los ésteres metílicos de ácidos grasos, condición necesaria para su posterior análisis por cromatografía gaseosa. Los ésteres metílicos fueron extraídos utilizando éter de petróleo (3 extracciones con $2 \mathrm{ml}$ de solvente c/u, desechando la fase etérea). La composición en ácidos grasos fue obtenida por cromatografía gaseosa utilizando una columna capilar de 50 metros de longitud y $0,25 \mathrm{~mm}$ de diámetro interno (CPSil 88, Chrompack, Holanda) en un cromatógrafo gaseoso Hewlett Packard 6890 con detector de ionización 
de llama. El perfil de ácidos grasos de cada una de las muestras se obtuvo por comparación de los tiempos de retención relativos de cada uno de ellos respecto de estándares de metilésteres de ácidos grasos de 99\% de pureza (NuCheck Prep. Inc) analizados previamente en la misma columna (30). A partir del perfil de ácidos grasos se calculó para la quinoa NM el índice de poliinsaturación (AGPI/AGS) y la relación n-6/n-3 de la fracción de grasa.

\subsection{Contenido total de saponinas}

La determinación del contenido total de saponinas en las semillas de quinoa estudiadas se llevó a cabo de acuerdo a la metodología descripta por Gianna y col. (31). La misma consistió en una extracción asistida con microondas y posterior cuantificación por derivatización mediante reacción de LibermannBurchard (ácido sulfúrico concentrado: anhídrido acético concentrado, 5:1) (32-34) y medición por espectrofotometría a $528 \mathrm{~nm}$ con un espectrofotómetro Perkin Elmer Lambda 35 utilizando ácido oleanólico para la curva de calibración.

\section{Análisis estadístico}

Todas las determinaciones se llevaron a cabo por duplicado. Se realizó un análisis estadístico descriptivo informando la media, el desvío estándar (DS), el rango de variación (valores mínimos y máximos) y el coeficiente de variación expresado como porcentaje (CV\%) para cada variable. Asimismo, el análisis comparativo de las medias se realizó por Test de Student y se consideraron significativas las probabilidades menores al 5\%. Para dichos análisis se utilizó el programa estadístico GraphPad Instant 3.01.

\section{RESULTADOS Y DISCUSIÓN}

En la Tabla 1 se presenta la composición centesimal, el perfil de ácidos grasos y el contenido de saponinas de los 4 genotipos de quinoa del nivel del mar (NM). Asimismo, la comparación de la composición centesimal entre NM y las quinoas provenientes del NOA se presenta en la Figura 1.

Con respecto al contenido proteico, el valor promedio de las quinoas del nivel del mar (Tabla 1),

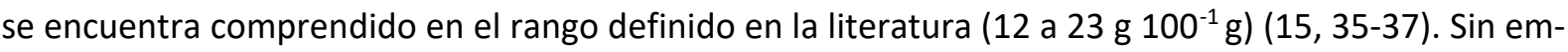
bargo, como se observa en la Fig. 1, el mismo fue estadísticamente menor con respecto al de la quinoa

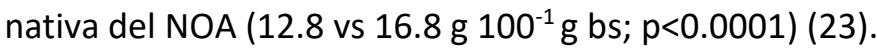

Por otra parte, el contenido promedio de FDT en las quinoas NM (Tabla 1) fue comparativamente mayor con respecto a los valores descriptos en la literatura para quinoas de Perú y Bolivia (1.9 - $5.7 \mathrm{~g}$ $\left.100 \mathrm{~g}^{-1}\right)(38-40)$. Estas diferencias podrían atribuirse a las diferentes metodologías para determinar el 
contenido de fibra. En general los datos de las tablas de composición de alimentos refieren a la determinación de fibra cruda, mientras que en el presente trabajo se determinó el contenido de FDT utilizando un método gravimétrico enzimático reconocido como la metodología más apropiada. Asimismo, los valores hallados para las quinoas del nivel del mar fueron similares a las del NOA (Fig 1), pero con un menor rango de variación (NM: 11.3 - 14.3; NOA: 9.8 - 16.0 g100-1 bs) (23).

En cuanto al contenido de cenizas, los genotipos del NM presentaron valores mayores (Tabla 1)

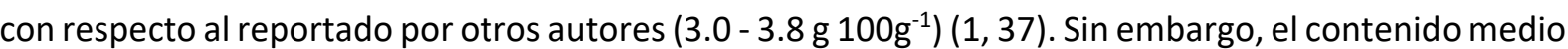
no fue estadísticamente diferente al observado en las quinoas del NOA (23) (Fig 1).

Asimismo, como se observa en la Tabla 1, el contenido promedio de grasa en las quinoas NM se

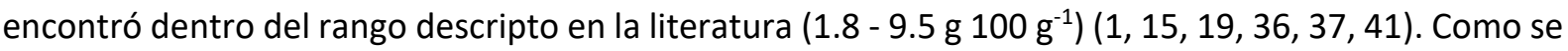
muestra en la Fig 1, tampoco se encontró diferencia significativa en relación al contenido graso con

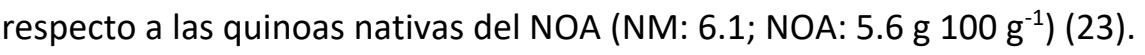

El perfil de los ácidos grasos de la quinoa NM se presenta en la Tabla 1 mientras que la comparación con respecto a las quinoas del NOA se muestra en la Figura 2. Los valores hallados tanto de ácidos grasos saturados (AGS), como de ácidos grasos monoinsaturados (AGM) y ácidos grasos poliinsaturados (AGPI) son similares a los reportados en la literatura, de 12 a 19\% (principalmente ácido palmítico), de 25 a 29\% (principalmente ácido oleico) y de 52 a 63\% (predominantemente ácido linoleico), respectivamente (15). Por el contario, como se observa en la Fig. 2, la quinoa NM mostró diferencias significativas con respecto a la quinoa nativa del NOA, tanto en el contenido medio de AGS totales como el de ácidos grasos insaturados (AGI) totales. En relación a los AGS mirístico, palmítico y esteárico, los valores hallados fueron estadísticamente menores que en la quinoa del NOA $(0.6 \%, 14.5 \%$ y $2.2 \%$ respectivamente, $p<0.01$ ) (23). Mientras que el valor medio de AGI en las quinoas NM representó el $87.8 \%$, en las del NOA fue del $83 \%$ ( $p<0.001$ ) (23). Esto se debe fundamentalmente al contenido significativamente mayor de ácido linoleico (C18:2 $n-6)$ en las quinoas NM con respecto a las del NOA (59.3\% vs $50.6 \% ; p<0.0001)$ (23), ya que el ácido linolénico (C18:3 n-3) no presentó diferencia significativa (NM: 6.2\% vs NOA: 7.1\%) (23); por el contrario, el contenido de ácido oleico (C18:1 n-9) fue significativamente menor que en las del NOA ( $21.3 \%$ vs $25.4 \%$; $p<0.001)(23)$.

De acuerdo con el perfil de ácidos grasos hallado en las quinoas NM, el índice AGPI/AGS fue de 5.4 y la relación n-6/n-3 fue de 9.9. Ambos valores están en concordancia con las recomendaciones dietéticas del National Research Council (42) que son AGPI/AGS> 1.5 y n-6/n-3=5-10. Al compararlos con los obtenidos para la quinoa nativa del NOA, el índice de poliinsaturación se presentó significativamente mayor (NM: 5.4 vs NOA: 3.6; $p<0.0001$ ), mientras que la relación $n-6 / n-3$ fue similar (NM: 9.9 vs NOA: 8.5) (23).

Finalmente, con respecto al contenido de saponinas de la quinoa NM (Tabla 1), los valores para los 4 genotipos se encontraron dentro del amplio rango reportado en la literatura para quinoa cruda 
$(0,1 \%$ a $5 \%$ p/p) $(6,10,37,43,44)$. Teniendo en cuenta la clasificación organoléptica del grano en base a su contenido de saponinas, dulce de 0,0 a $0,1 \%$, semi-dulce de 0,1 a $1 \%$ y amarga de 1 a $3 \%$ (45), se puede clasificar a la quinoa NM como quinoa amarga. Los contenidos hallados fueron similares a los de la quinoa del NOA (NM: $1.6-2.6 \%$ vs NOA: $1.2-3.2 \%)(23)$.

\section{CONCLUSIONES}

Este estudio permitió caracterizar los atributos nutricionales de diferentes genotipos de quinoa del nivel del mar cultivados en ambiente templado de Argentina y de esta manera contribuir a los datos nacionales de quinoa en las tablas de composición de alimentos. Los resultados del mismo sugieren que el genotipo y el ambiente de cultivo incidirían en las propiedades nutricionales de la quinoa, lo que debería ser tenido en cuenta para su elección y aprovechamiento desde el punto de vista nutricional. En comparación con las quinoas nativas del noroeste argentino, las del nivel del mar poseen un contenido menor de proteínas y mayor de ácidos grasos poliinsaturados, encontrándose ambas en los rangos reportados en la literatura internacional. La incorporación de este grano en las dietas de los consumidores o en programas y políticas relacionadas con la nutrición podría mejorar la biodiversidad de la dieta occidental que caracteriza a la Argentina. Por lo tanto, su cultivo se presentaría como una alternativa válida a los cereales en la región pampeana.

\section{REFERENCIAS BIBLIOGRÁFICAS}

1. Vega-Galvez, A.; Miranda, M.; Vergara, J.; Uribe, E.: Puente, L.; Martínez, E.A., 2010. Nutrition facts and functional potential of quinoa (Chenopodium quinoa Willd.), an ancient andean grain: a review. J. Sci. Food Agric. 90, 2541-2547.

2. Jacobsen, S.E.; Mujica, A.; Jensen, C.R., 2003. The resistance of quinoa (Chenopodium quinoa Willd.) to adverse abiotic factors. Food Rev. Int. 19, 1-2: 99-109.

3. Stikic, R.; Glamoclija, D.; Demin, M.; Vucelic-Radovic, B.; Jovanovic, Z.; Milojkovic-Opsenica, D.; Jacobsen, S.E.; Milovanovic, M., 2012. Agronomical and nutritional evaluation of quinoa seeds (Chenopodium quinoa Willd.) as an ingredient in bread formulations. J. Cereal Sci. 55, 132-138.

4. Nowak, V.; Du, J.; Charrondière, R. 2016. Assessment of the nutritional composition of quinoa (Chenopodium Quinoa Willd.). Food Chem. 193, 47-54.

5. Wilson H.D. (1990) Quinua and relatives (Chenopodium sect. Chenopodium subsect. Cellulata). Econ. Bot. 44, 3: 92-110

6. Valencia-Chamorro, S.A., 2003. Quinoa. In: Caballero B. (Ed.) Encyclopedia of food science and nutrition, vol 8. Academic Press. (EEUU), 4895-4902. 
7. Food and Agriculture Organization, 2011. Technical Report: Quinoa: an ancient crop to contribute to world food security. Rome. Regional office for Latin America and the Caribbean.

8. Curti, R.N.; Andrade, A.J.; Bramardi, S.; Velasquez, B.; Bertero, H.D., 2012. Ecogeographic structure of phenotypic diversity in cultivated populations of quinoa from Northwest Argentina. Ann. App. Biol. 160, 114-125.

9. Tapia, M.E., 2014.El largo camino de la quinoa: ¿quiénes escribieron su historia?. En: Bazile, D. et al. (Eds.), "Estado del arte de la quinua en el mundo en 2013". FAO (Chile) y CIRAD, (Francia), 310.

10. Tapia, M.E., 2000 Agronomía de los cultivos andinos. Granos andinos: Quinoa. En: Izquierdo, J. et al. (Eds.), “Cultivos andinos subexplotados y su aporte a la alimentación”. FAO (Chile). Disponible en: http://www.fao.org/tempref/GI/Reserved/FTP_FaoRlc/old/prior/segalim/prodalim /prodveg/cdrom /contenido/libro10/home10.htm

11. Bertero, H.D.; King, R.W.; Hall A.J., 1999. Modelling photoperiod and temperature responses of flowering in quinoa (Chenopodium quinoa W.). Field Crops Res. 63, 19-34.

12. Ruales, J.; Nair, B.M., 1992. Nutritional quality of the protein in quinoa (Chenopodium quinoa, Willd.) seeds. Plant Foods Hum. Nutr. 42, 2: 1-11.

13. Repo-Carrasco, R.; Espinoza, C.; Jacobsen, S.E., 2003. Nutritional value and use of the Andean crops quinoa (Chenopodium quinoa) and kañiwa (Chenopodium pallidicaule). Food Rev. Int. 19, 1-2: 179-189.

14. Wijngaard, H.H.; Arendt, E.K., 2006. Buckwheat. Cereal Chem. 8, 391-401.

15. Abugoch James, L.E., 2009. Quinoa (Chenopodium quinoa Willd.): composition, chemistry, nutritional, and functional properties. Adv. Food. Nutr. Res. 58, 1-31.

16. Haros, C. M.; Schoenlechner, R., 2017. Pseudocereals: Chemistry and technology. Wiley-Blackwell (eds) First ed. (UK). Disponible en: https://doi.org/10.1002/9781118938256

17. Vilcacundo, R.; Hernández-Ledesma, B., 2017. Nutritional and biological value of quinoa (Chenopodium quinoa Willd.). Curr. Opin. in Food Sci., 14, 1-6.

18. Mujica, A.; Izquierdo, J.; Marathee J.P.; 2001. Origen y descripción de la quinua. En: Mujica A., et al. (Eds.), "Quinua: Ancestral cultivo andino, alimento del presente y futuro". FAO. UNA. CIP. (Chile) 26-59.

19. Bhargava, A.; Shukla, S.; Ohri, D., 2007. Evaluation of foliage yield and leaf traits in Chenopodium spp. in multiyear trials. Euphytica 153, 3: 199-213.

20. Repo-Carrasco-Valencia, R.A.; Encina, C.R.; Binaghi, M.J.; Greco, C.B.; Ronayne de Ferrer, P.A., 2010. Effects of roasting and boiling of quinoa, kiwicha and kañiwa on composition and availability of minerals in vitro. J. Sci. Food Agric. 90, 2068-2073. 
21. González, J.A.; Konishi, Y.; Bruno M.; Valoy M.; Prado F.E., 2011. Interrelationships among seed yield, total protein and amino acid composition of ten quinoa (Chenopodium quinoa) cultivars from two different agroecological regions. J. Agric. Food Chem. 92, 1222-1229.

22. Miranda, M.; Vega-Galvez, A.; Martínez, E.A.; Lopez, J.; Marin, R.; Aranda, M.; Fuentes, F., 2013. Influence of contrasting environments on seed composition of two quinoa genotypes: nutritional and functional properties. Chil. J. Agric. Res. 73, 2: 108-116.

23. Vidueiros, S.M.; Curti, R.N.; Dyner, L.M.; Binaghi, M.J.; Peterson, G.; Bertero, H.D.; Pallaro, A.N., 2015. Diversity and interrelationships in nutritional traits in cultivated quinoa (Chenopodium quinoa Willd) from Northwest Argentina. J. Cereal Sc. 62, 87-93.

24. De Santis, G.; D'Ambrosio, T.; Rinaldi, M.; Rascio, A., 2016. Heritabilities of morphological and quality traits and interrelationships with yield in quinoa (Chenopodium quinoa Willd.) genotypes in the Mediterranean environment. J. Cereal Sci, 70, 177-185.

25. Aluwi, N. A.; Murphy, K. M.; Ganjyal, G. M., 2017. Physicochemical characterization of different varieties of quinoa. Cereal Chem. 94, 5: 847-856.

26. Bertero, H.D., 2001. Quinoa (Chenopodium quinoa, Chenopodiaceae) puede llegar a ser un cultivo importante para la agricultura argentina. Bol. Soc. Argent. Bot. 36, 3-4: 309-314.

27. Bertero, H.D.; de la Vega, A.J.; Correa, G.; Jacobsen, S.E.; Mujica, A., 2004. Genotype and genotypeby-environment interaction effects for grain yield and grain size of quinoa (Chenopodium quinoa Willd.) as revealed by pattern analysis of international multi-environment trials. Field Crops Res. 89, 299-318.

28. Association of Official Analytical Chemist, 2000 Official Methods of Analysis of AOAC International, seventeenth ed., vol. I. y II. Inc. Gaithersburg, Maryland, USA.

29. Folch, J.; Lees, M.; Sloane Stanley, Gh., 1957. A simple method for isolation and purification of total lipids from animal tissue. J. Biol. Chem. 226, 1: 497-509

30. Tavella, M.; Peterson, G.; Espeche, M.; Cavallero, E.; Cipolla, L.; Perego, L.; Caballero, B., 2000. Trans fatty acid content of a selection of foods in Argentina. Food Chem. 69, 209-213.

31. Gianna, V.; Montes, J.M.; Calandri, E.L.; Guzmán, C.A. 2012. Impact of several variables on the microwave extraction of Chenopodium quinoa Willd saponins. Int. J. Food Sci. Tech. 47, 15931597.

32. Abisch, E.; Reichstein, T., 1960. Orientierende chemische untersuchung einiger Apocynaceen. Helv. Chim. Acta 43, 6: 1844-1861

33. Hostettmann, K.; Marston, A. 2005. Saponins. Cambridge University Press. (EEUU).

34. Monje, C.; Yarko, A; Raffaillac, J.P. 2006. Determinación de saponina total en quinoa (Chenopodium quinoa Willd) Memoria IV Congreso Nacional de la Asociación Boliviana de Protección Vegetal. Abril. C.E.A.C. - Dpto. Fitotecnia-FCAPV-UTO. ABPV. Oruro, Bolivia. 
35. Koziol, M.J., 1992. Chemical composition and nutritional Evaluation of quinoa (Chenopodium quinoa Willd.). J. Food Compost. Anal. 5, 35-68

36. Ruales, J.; Nair, B.M., 1993. Content of fat, vitamins and minerals in quinoa (Chenopodium quinoa Willd.) seeds. Food Chem. 48, 2: 131-136

37. Miranda, M.; Vega-Gálvez, A.; Quispe-Fuentes, I.; Rodríguez, M.J.; Maureira, H.; Martínez, E.; 2012. Nutritional aspects of six quinoa (Chenopodium quinoa Willd.) ecotypes from three geographical areas of Chile. Chil. J. Agric. Res. 72, 2: 175-181.

38. Mejía, L.; Guillén, E.; Silberstein, M.; Mendoza, A.; Vidal, F.; Cherroni, D.; de la Oliva, O., 2005. Cereales y derivados. En: Tabla Boliviana de composición de alimentos (4ae ed.). La Paz. Bolivia.: Ministerio de Salud y Deportes.

39. Reyes García, M.; Gómez-Sánchez Prieto, I.; Espinoza Barrientos, C.; Bravo Rebatta, F.; Ganoza Morón, L., 2009. Cereales y derivados. En: Tablas peruanas de composición de alimentos (8aed.). Lima: Ministerio de Salud, Instituto Nacional de Salud.

40. FAO/Latin Food, 2009. Tabla de composición de alimentos de América Latina. Disponible en: http://www.rlc.fao.org/es/conozca-fao/que-hace-fao/estadisticas/composicion-alimentos

41. Koziol, M.J., 1993. Quinoa: a potential new oil crop. En: Simonm, J.E.; Janick, J. (Eds.) New crops. Wiley (EEUU), 328-336.

42. National Research Council, 2005. Dietary Reference Intakes for energy, carbohydrates, fiber, fat, fatty acids, cholesterol, protein, and amino acids (macronutrients). The National Academies Press, Washington, DC.

43. Stuardo, M.; San Martin, R., 2008. Antifungal properties of quinoa (Chenopodium quinoa Willd.) alkali treated saponins against Botrytis cinerea. Ind. Crops Prod. 27, 296-302.

44. Jancurová, M.; Minaravičova, L.; Dandar, A., 2009. Quinoa - A review. Czech. J. Food Sci. 27, 2: $71-$ 79.

45. Güçlü-Üstündağ, O; Mazza, G., 2007. Saponins: properties, applications and processing. Crit. Rev. Food Sci. Nutr. 47, 3: 231-258. 
Tabla 1. Composición centesimal, perfil de ácidos grasos y contenido de saponinas de quinoa del nivel del mar

\begin{tabular}{|c|c|c|c|c|c|c|c|c|}
\hline & \multicolumn{4}{|c|}{ Genotipos } & \multirow[b]{3}{*}{ Promedio } & \multirow[b]{3}{*}{ Rango } & \multirow[b]{3}{*}{$D^{d}$} & \multirow[b]{3}{*}{ CV (\%) } \\
\hline & CO-407 & Salto de Agua & NL-6 & 2-Want & & & & \\
\hline & & & & & & & & \\
\hline Humedad $^{a}$ & 10.8 & 9.6 & 8.9 & 9.7 & 9.7 & $8.9-9.6$ & 0.8 & 8.6 \\
\hline Proteínas $^{\mathrm{a}}$ & 13.0 & 12.4 & 13.6 & 12.3 & 12.8 & $12.3-13.6$ & 1.4 & 11.3 \\
\hline Cenizas $^{a}$ & 5.4 & 4.9 & 4.7 & 4.4 & 4.9 & $4.4-5.4$ & 0.8 & 16.8 \\
\hline Fibra Dietaria Totala & 14.3 & 11.6 & 11.4 & 11.3 & 12.1 & $11.3-14.3$ & 2.4 & 19.8 \\
\hline Carbohidratos $^{\mathrm{a}}$ & 61.9 & 65.0 & 64.8 & 65.0 & 64.2 & $61.9-65.0$ & 3.0 & 4.7 \\
\hline Grasa $^{a}$ & 5.4 & 6.0 & 6.0 & 7.0 & 6.1 & $5.4-7.0$ & 0.9 & 14.7 \\
\hline AGS $^{b}$ totales & 12.6 & 12.6 & 11.2 & 12.4 & 12.2 & $11.2-12.6$ & 1.2 & 10.0 \\
\hline C14:0 $0^{b}$ & 0.2 & 0.4 & 0.3 & 0.3 & 0.3 & $0.2-0.4$ & 0.1 & 29.2 \\
\hline $\mathrm{C} 16: 0^{\mathrm{b}}$ & 11.5 & 11.5 & 10.3 & 10.7 & 11.0 & $10.3-11.5$ & 0.9 & 8.4 \\
\hline $\mathrm{C} 18: 0^{\mathrm{b}}$ & 1.0 & 0.8 & 0.8 & 1.4 & 1.0 & $0.8-1.4$ & 0.5 & 50.7 \\
\hline $\mathrm{AGI}^{\mathrm{b}}$ totales & 87.4 & 87.4 & 88.8 & 87.6 & 87.8 & $87.4-88.8$ & 1.2 & 1.4 \\
\hline C18:1 n-9 & 19.3 & 20.3 & 22.7 & 22.8 & 21.3 & $19.3-22.8$ & 2.5 & 11.6 \\
\hline$C 18: 2 n-6^{b}$ & 60.7 & 60.7 & 57.7 & 58.1 & 59.3 & $57.7-60.7$ & 2.9 & 4.9 \\
\hline C18:3 n-3 ${ }^{b}$ & 6.9 & 5.6 & 6.7 & 5.6 & 6.2 & $5.6-6.9$ & 1.0 & 16.2 \\
\hline Saponinas ${ }^{c}$ & 1.6 & 2.0 & 2.6 & 2.4 & 2.1 & $1.6-2.6$ & 1.0 & 44.9 \\
\hline
\end{tabular}

${ }^{\mathrm{a}} \mathrm{g} 100 \mathrm{~g}^{-1} \mathrm{bs}$

${ }^{\mathrm{b}} \mathrm{g} 100 \mathrm{~g}^{-1}$ del total de ácidos grasos (AGS totales es la suma de C14:0, C16:0 y C18:0; mientras que AGI totales corresponde a C18:1n-9, C18:2n-6, C18:3n-6 y C18:3n- ${ }^{3}$

${ }^{c} \mathrm{~g} 100 \mathrm{~g}^{-1}$

${ }^{d} D S$ : Desviación estándar

${ }^{\mathrm{f}} \mathrm{CV}(\%)$ : Coeficiente de variación 


\section{Composición Centesimal}

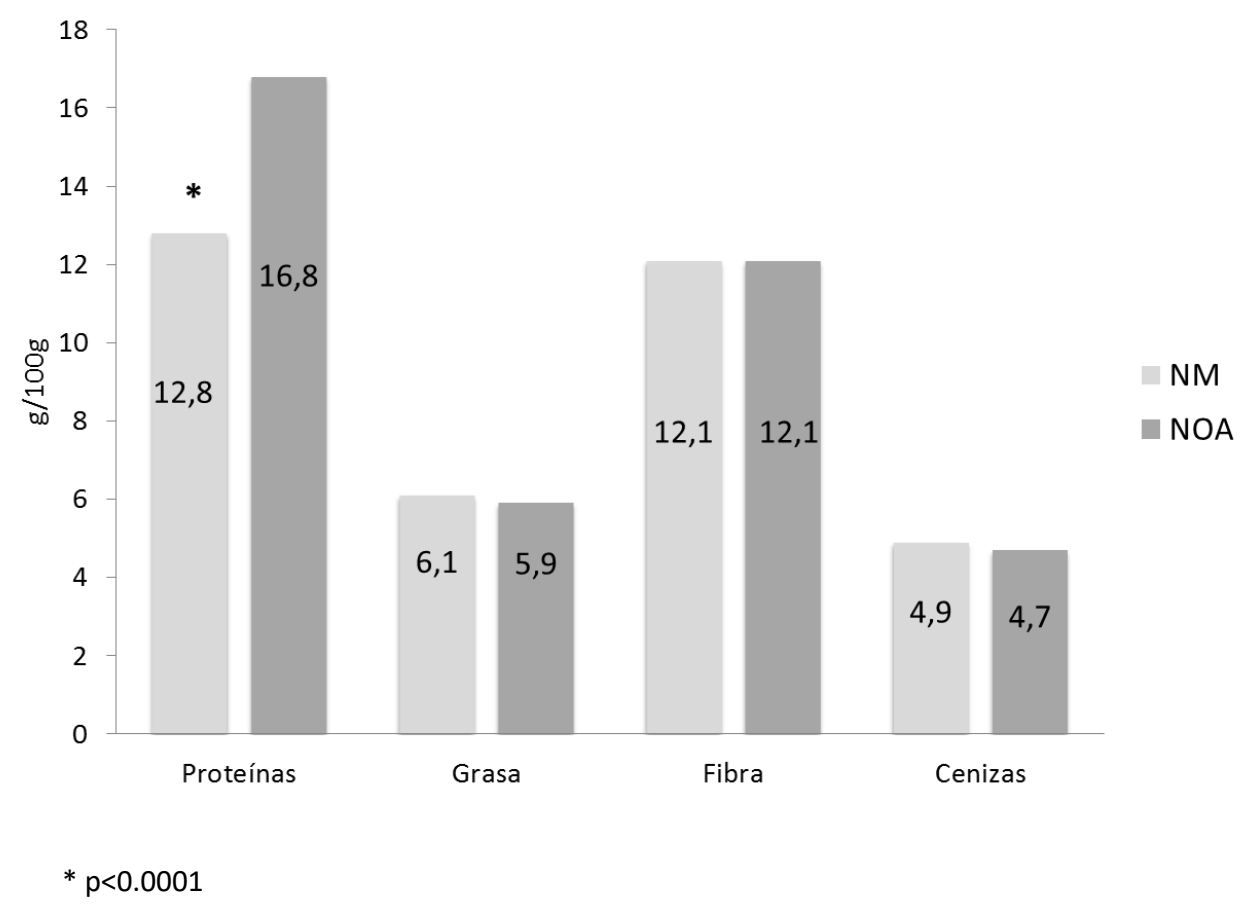

Figura 1. Composición centesimal de quinoa del nivel del mar versus quinoa del noroeste argentino

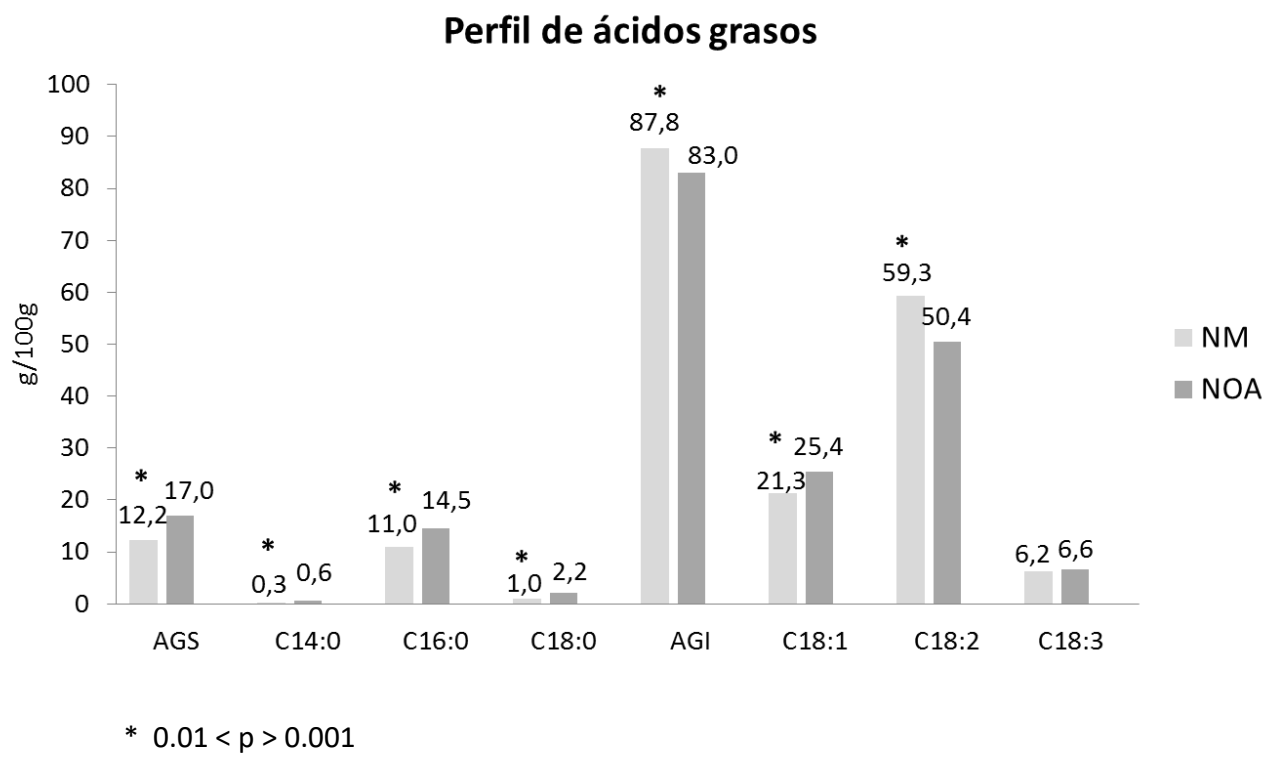

Figura 2. Perfil de ácidos grasos de quinoa del nivel del mar versus quinoa del noroeste argentino 